\title{
Experimentally testing the role of blood vessels in the full scattering profile: solid phantom measurements
}

\author{
Idit Feder, Hamootal Duadi, Moti Fridman, Tamar Dreifuss, and Dror Fixler \\ Faculty of Engineering and the Institute of Nanotechnology and Advanced Materials, Bar Ilan University, Israel \\ e-mail: Dror.Fixler@biu.ac.il
}

\begin{abstract}
Optical methods for biomedical purposes mostly use reflected light, while few of them use the transmitted light. The blood vessels pose a challenge to these methods due to their high absorption and scattering coefficients as well as their change in size during respiration, and they are also naturally distributed in size within the body and between individuals. We suggest the full scattering profile (FSP) method in order to investigate the light at every possible exit angle. Our model of FSP successfully describes the role of the blood vessel diameter in light-tissue interaction. By means of the new point of view of FSP, we found the isobaric point, which is non-dependent on the optical properties. The uniqueness of the isobaric point is that it overcomes the shielding effect, which has known influence on the reflected light, for various vascular diameters of the same volume. We present these findings experimentally by measuring cylindrical phantoms with blood vessels in different diameters, and compare the results to our simulation results. The importance of the immunity to the shielding effect is that it allows self-calibration in clinical measurements and decreases the calibration error. In addition, by using the isobaric point we can cope with changes in blood vessel diameters and not assume microcirculation only. (c) 2016 Journal of Biomedical Photonics \& Engineering.
\end{abstract}

Keywords: absorption, light impulse, biophotonics, medical physics.

Paper \#3096 received 2016.09.15; revised manuscript received 2016.10.28; accepted for publication 2016.10.31; published online 2016.12.23. doi: 10.18287/JBPE16.02.040301

\section{References}

1. S. L. Jacques, "Optical properties of biological tissues: a review," Physics in medicine and biology 58(11), R37 (2013).

2. B. Xiong, L. Peng, X. Cao, Y. He, and E. S. Yeung, "Optical analysis of biological hydrogen sulphide: an overview of recent advancements," Analyst 140(6), 1763-1771 (2015).

3. D. I. Ellis, D. P. Cowcher, L. Ashton, S. O'Hagan, and R. Goodacre, "Illuminating disease and enlightening biomedicine: Raman spectroscopy as a diagnostic tool," Analyst 138(14), 3871-3884 (2013).

4. R. Ankri, H. Taitelbaum, and D. Fixler, "Reflected light intensity profile of two-layer tissues: phantom experiments," Journal of biomedical optics 16(8), 085001, (2011).

5. D. Fixler, and R. Ankri, "Subcutaneous gold nanorods detection with diffusion reflection measurement," Journal of biomedical optics 18(6), 061226 (2013).

6. R. A. J. Groenhuis, J. J. Ten Bosch, and H. A. Ferwerda, "Scattering and absorption of turbid materials determined from reflection measurements. 2: Measuring method and calibration," Applied optics 22(16), 24632467 (1983).

7. D. Jakubowski, F. Bevilacqua, S. Merritt, A. Cerussi, and B. J. Tromberg, "Quantitative absorption and scattering spectra in thick tissues using broadband diffuse optical spectroscopy," Chapter 12 in Biomedical Optical Imaging, J. G. Fujimoto, and D. L. Farkas (eds.), Oxford University Press, New York, 330-355 (2009).

8. T. H. Pham, O. Coquoz, J. B. Fishkin, E. Anderson, and B. J. Tromberg, "Broad bandwidth frequency domain instrument for quantitative tissue optical spectroscopy," Review of Scientific Instruments 71(6), 2500-2513, (2000). 
9. M. Friebel, J. Helfmann, U. Netz, and M. Meinke, "Influence of oxygen saturation on the optical scattering properties of human red blood cells in the spectral range 250to2000nm," Journal of biomedical optics 14(3), 034001 (2009).

10. M. Friebel, A. Roggan, G. Müller, and M. Meinke, "Determination of optical properties of human blood in the spectral range 250to1100nm using Monte Carlo simulations with hematocrit-dependent effective scattering phase functions," Journal of biomedical optics 11(3), 034021 (2006).

11. L. Zhang, A. Shi, and H. Lu, "Determination of optical coefficients of biological tissue from a single integratingsphere," Journal of Modern Optics 59(2), 121-125 (2012).

12. I. Feder, H. Duadi, and D. Fixler, "Experimental system of the full scattering profile of circular phantoms," Biomed. Opt. Express 6(8), 2877-2886 (2015).

13. H. Duadi, I. Feder, and D. Fixler, "Linear dependency of full scattering profile isobaric point on tissue diameter," Journal of biomedical optics 19(2), 026007 (2014).

14. H. Duadi, D. Fixler, and R. Popovtzer, "Dependence of light scattering profile in tissue on blood vessel diameter and distribution: a computer simulation study," Journal of biomedical optics 18(11), 111408 (2013).

15. H. Duadi, and D. Fixler, "Influence of multiple scattering and absorption on the full scattering profile and the isobaric point in tissue," Journal of biomedical optics 20(5), 056010 (2015).

16. I. Feder, H. Duadi, T. Dreifuss, and D. Fixler, "The influence in the full scattering profile from cylindrical tissues following changes in vessels diameter: experimental evidence for the shielding effect," Journal of biophotonics 9(10), 1001-1008 (2015).

17. L. O. Svaasand, E. J. Fiskerstrand, G. Kopstad, L. T. Norvang, E. K. Svaasand, J. S. Nelson, and M. W. Berns, "Therapeutic response during pulsed laser treatment of port-wine stains: dependence on vessel diameter and depth in dermis," Lasers in Medical Science 10(4), 235-243 (1995)."

18. H. Liu, B. Chance, A. H. Hielscher, S. L. Jacques, and F. K. Tittel, "Influence of blood vessels on the measurement of hemoglobin oxygenation as determined by time-resolved reflectance spectroscopy," Medical physics 22(8), 1209-1217 (1995).

19. S. L. Jacques, "Optical assessment of cutaneous blood volume depends on the vessel size distribution: a computer simulation study," Journal of biophotonics 3(1-2), 75-81 (2010).

20. W. Verkruysse, G. W. Lucassen, J. F. de Boer, D. J. Smithies, J. S. Nelson, and M. J. C. van Gemert, "Modelling light distributions of homogeneous versus discrete absorbers in light irradiated turbid media," Physics in Medicine and Biology 42(1), 51-65 (1997).

21. M. Firbank, E. Okada, and D. T. Delpy, "Investigation of the effect of discrete absorbers upon the measurement of blood volume with near-infrared spectroscopy," Physics in medicine and biology 42(3), 465-477 (1997).

22. A. Talsma, B. Chance, and R. Graaff, "Corrections for inhomogeneities in biological tissue caused by blood vessels," J. Opt. Soc. Am. 18(4), 932-939 (2001).

23. K. K. Tremper,’Pulse oximetry," Chest 95(4), 713-715 (1989).

24. H. Duadi, M. Nitzan, and D. Fixler, "Simulation of oxygen saturation measurement in a single blood vein," Opt. Lett. 41(18), 4312-4315 (2016).

25. M. Nitzan, S. Noach, E. Tobal, Y. Adar, Y. Miller, E. Shalom, and S. Engelberg, "Calibration-Free Pulse Oximetry Based on Two Wavelengths in the Infrared — A Preliminary Study," Sensors 14(4), 7420-7434 (2014).

26. V. V. Tuchin, A. N. Bashkatov, E. A. Genina, V. I. Kochubey, V. V. Lychagov, S. A. Portnov, N. A. Trunina, D. R. Miller, S. Cho, H. Oh, B. Shim, M. Kim, J. Oh, H. Eum, Y. Ku, D. Kim, and Y. Yang, "Finger tissue model and blood perfused skin tissue phantom," Proc. SPIE 7898, 78980Z (2011).

27. R. Ankri, H. Taitelbaum, and D. Fixler, "On Phantom experiments of the photon migration model in tissues," The Open Optics Journal 5(1), 28-32 (2011).

28. J. S. Dam, C. B. Pedersen, T. Dalgaard, P. E. Fabricius, P. Aruna, and S. Andersson-Engels, "Fiber-optic probe for noninvasive real-time determination of tissue optical properties at multiple wavelengths," Applied optics 40(7), 1155-1164 (2001).

29. I. Feder, M. Wróbel, H. Duadi, M. Jędrzejewska-Szczerska, and D. Fixler, "Experimental results of full scattering profile from finger tissue-like phantom," Biomed. Opt. Express 7(11), 4695-4701 (2016).

\section{Introduction}

In recent years, optical methods are very useful in the detection of various physiological states. They are noninvasive and rely on the minor changes in the optical properties during disease or in other physiological states [1-3]. Most of the optical methods for biological applications use the reflected light [4-8], while few of them use the transmitted light [9-11].

In a previous work, we presented our new system for measuring the full scattering profile (FSP) of cylindrical tissues [12]. We suggested this method of measuring the ejected light from every possible exit angle as a new point of view for light-tissue interactions. In order to investigate the influence of optical properties and blood 
vessel diameters on the light scattering, we simulated the FSP of cylindrical tissues using a Monte Carlo simulation [13-15], and measured liquid and solid phantoms [16] with different reduced-scattering coefficients $\left(\mu_{s}^{\prime}\right)$ and with blood vessels in different diameters, prepared in our lab.

The main findings that we published are the isobaric point and the shielding effect in the FSP. The isobaric point is a unique point in the FSP that is independent on the optical properties of the tissue. This point, from a clinical perspective, can be a self-reference point for extracting optical properties. The angle of the isobaric point linearly depends on the tissue diameter, as we have validated both in simulation and experimentally [12, 13].

The second stage of the research focused on heterogeneous tissue-like phantoms that included blood vessels. By investigating the blood vessel diameter's influence on the FSP, we presented in a simulation as well as an experimentally, the shielding effect in the FSP. The meaning of the shielding effect is that the inner part of large vessels is not exposed to light and therefore is less effective in the determination of the optical path length.

The shielding effect, which was examined while measuring the reflected light, has been already discussed in literature by simulations [17] as well as experiments [18]. As Jacques [19] published, the shielding effect is significant proportionally to the blood vessel diameter (D) and to the blood absorption coefficient $\left(\mu_{a}\right)$. Verkruysse et al. [20] concluded that for $\mu_{a} D>1$, the shielding effect is more significant. Hence, for tissue optical properties in near infrared (NIR) the shieldingeffect does not occur under $1 \mathrm{~mm}$ vessel diameter. Others [21] claim that it is limited to a lower vessel diameter, and the shielding effect is significant for $\mu_{a} D>0.06$.

In other words, as Talsma [22] published, the apparent coefficients of blood vessels and the coefficients inside the blood vessels are the same for small values of $\mu_{t} D$ only (where $\left.\mu_{t}=\mu_{s}^{\prime}+\mu_{a}\right)$. However, for values higher then $\mu_{t} D$, the apparent absorption coefficient and the apparent reducedscattering coefficient are both smaller than the coefficients inside the blood vessels.

In a previous work, we discussed the shielding effect in the FSP of cylindrical silicon-based phantoms with blood vessels larger than $1 \mathrm{~mm}$. Due to a fabrication limit, $1 \mathrm{~mm}$ was the smallest diameter. Therefore, we are interested in obtaining smaller blood vessels in a different way, using narrowed glass capillaries (i.e. tapered capillaries) and liquid phantoms. Through this method, the glass volume remains constant in the phantom, even though the diameter is different. These tapered diameters are closer to the vascular system in human fingers.

The common method for measuring oxygen saturation in blood is pulse oximetry which is based on reflection of two wavelengths [23]. Since the optical properties of the tissue are depended on wavelength, the optical path length is different for the two wavelengths even though they advanced in the same surroundings. While the usage of nearby wavelengths, where the optical ways are similar, it is more challenging to extract the $\mathrm{SpO}_{2}$, due to the proximity of the extinction coefficients [24]. Therefore, the use of two light sources requires calibration, thus adding an error which is too high for some clinical problems [25]. Hence, it is necessary to consider the shielding effect for each one of them respectively.

In this paper, we present our system's sensitivity to vessels of a diameter less than $1 \mathrm{~mm}$. The isobaric point is still discernible in these small diameters. In addition, the prevailing investigation of the blood vessel diameter's influence on the light propagation is at the reflectance region. There we can see changes for the same blood volume that is placed in different vessel diameters, as well as at the transmission area. The uniqueness of our method is the isobaric point which is discovered to be immune to the shielding effect. We can use the isobaric point as a self-reference point for extracting optical properties in clinical applications, even though the blood vessels change their size during respiration, as they do in various physiological conditions. Therefore, it can be useful for more accurate blood perfusion measurements and blood oxygen saturation determination.

\section{Preparing of phantoms}

In order to simulate a skin tissue with blood vessels in different diameters, we tapered glass capillaries [26] using a glass processing unit (LZM-100, LAZERMaster) which is shown in Fig. 1. We designed the capillaries with different inner diameters per our requirements (750 $\mu \mathrm{m}, 600 \mu \mathrm{m}$ and $510 \mu \mathrm{m})$, as shown by the CT scan images in Fig. 2.

The operation principle of the LZM-100 machine is based on pulling both ends of the capillary while heating it with a CO2 laser system. The speed and heating power are programmable and since the machine is designed to work also with fiber, its accuracy is at the sub-micron level.

For the tissue around the blood vessels, we prepared phantoms using Intralipid 20\% (Lipofundin MCT/LCT $20 \%$, B. Braun Melsungen AG, Germany) as a scattering component $\left(\mu_{s}^{\prime}=1.04 \mathrm{~mm}^{-1}\right)$, and Agarose powder (SeaKem, LE Agarose, Lonza, USA), in order to convert the solution into gel [27]. We injected liquid phantoms that mimic blood in their scattering and absorption properties into the capillaries. As an absorbing component we used Indian ink $\left(\mu_{a}=1 \mathrm{~mm}^{-1}\right.$, $\mu_{s}^{\prime}=3 \mathrm{~mm}^{-1}$ ).

After the capillaries were filled with the blood-like solution, they were placed in a tube (13 $\mathrm{mm}$ diameter), as presented in Fig. 3. Finally, the background solution was added, in order to prevent cutting of the phantom after it stabilization and thus allowing the entry of undesirable air to the sample. The volume of the inner part of the capillaries in the different tubes remains constant (5\%) as the blood volume in human finger [19]. 


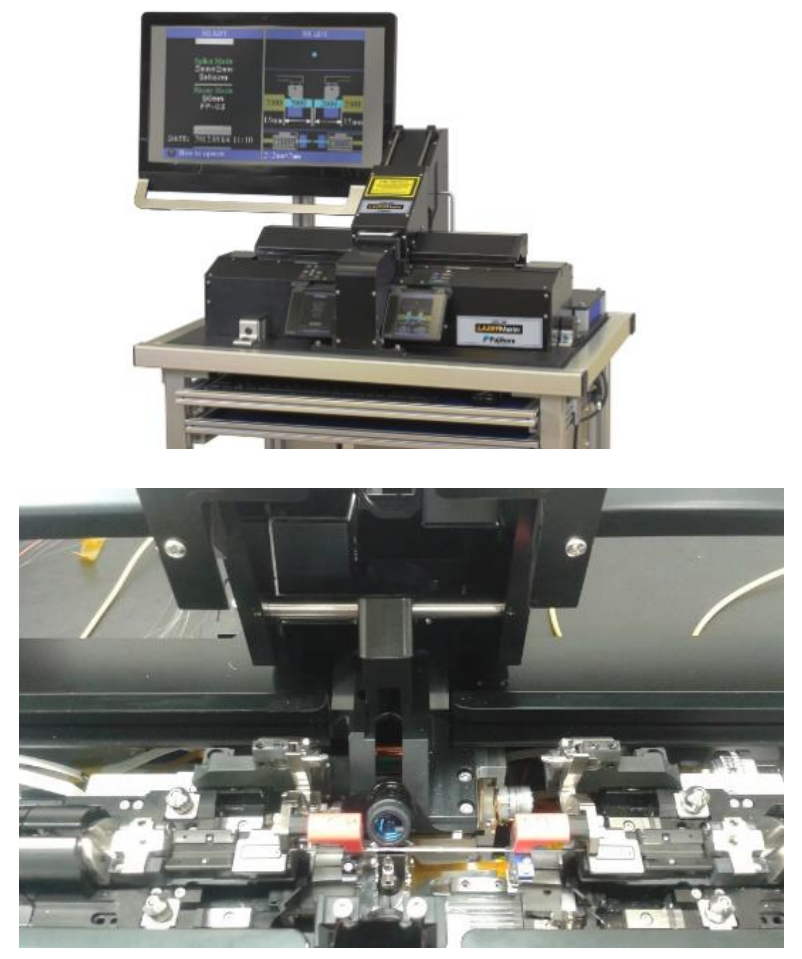

Fig. 1 (top) The LZM-100 system and (bottom) a capillary lying inside the LZM-100.

(a)

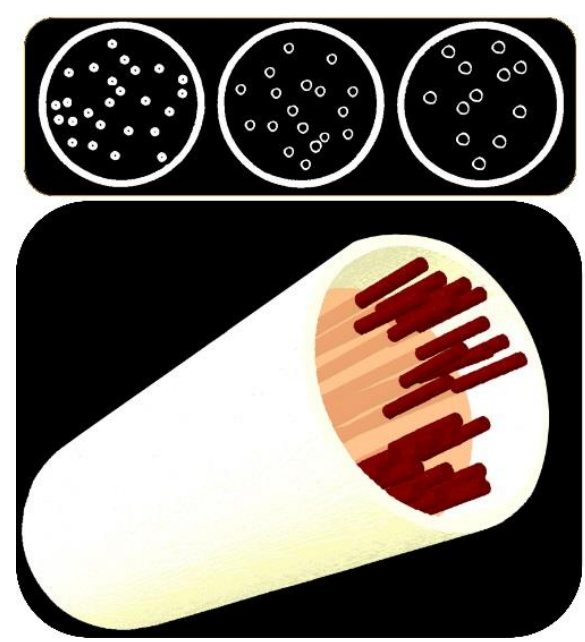

Fig. 2 CT scans of the capillaries in a $13 \mathrm{~mm}$ tube. Upper panel - 2D images: Tapered capillaries with inner diameter of (a) $510 \mu \mathrm{m}$ (b) $600 \mu \mathrm{m}$ and (c) 750 $\mu \mathrm{m}$. Lower panel - 3D image where tapered capillaries have an inner diameter of $510 \mu \mathrm{m}$.

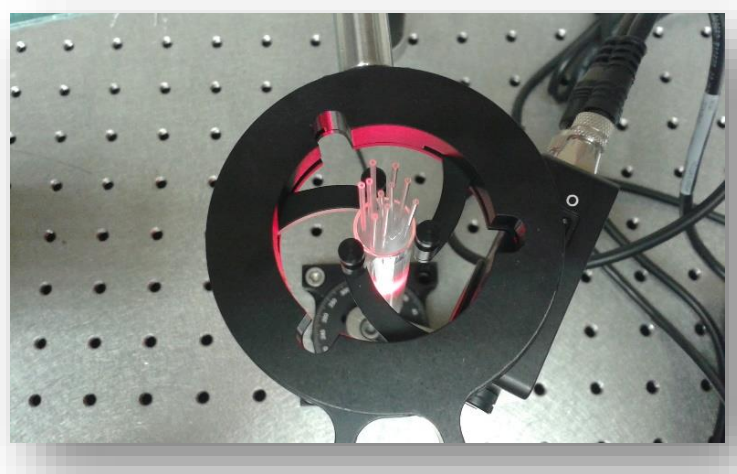

Fig. 3 Top view image of capillaries in a tube fixed in the FSP setup.

\section{Optical setup description}

We measured the phantoms, using our FSP optical system (Fig. 4). The set-up includes a He-Ne gas laser (Thorlabs, Newton, USA) with an excitation wavelength of $\lambda=632.8 \mathrm{~nm}$ and maximum power of $5 \mathrm{~mW}$. The waist of the laser beam is $\omega_{0}=0.63 \mathrm{~mm}$. We use a portable fixed gain silicon detector as a photo detector (PD). The PD has an active area of $0.8 \mathrm{~mm}^{2}$ and it is placed on a rotation stage in order to enable the full scattering profile measurements. The stage can be rotated around a tube with an accuracy of $1^{\circ}$. The voltage is measured every 5 degrees, when $0^{\circ}$ is the full transmission and $180^{\circ}$ is the full reflection. It should be noted that the light intensities from $145^{\circ}$ to $180^{\circ}$ were not measured, because of a system limitation; the PD's size blocks the laser. For each phantom, we repeated the measurements for different locations of the capillaries, in relation to the light source. The standard deviation due to capillaries' placement is presented in the results.

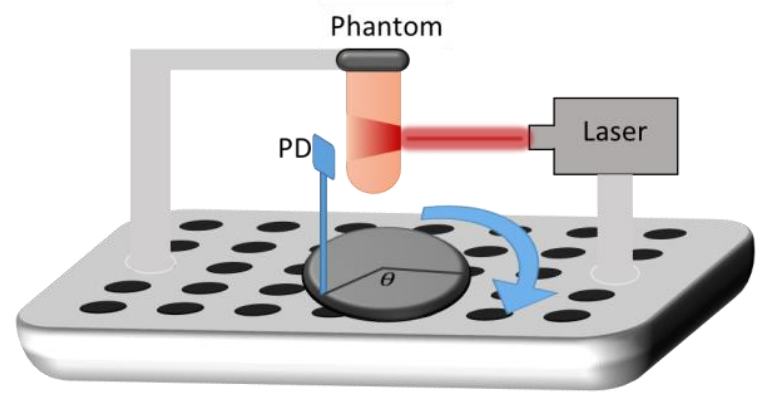

Fig. 4 A scheme of the FSP optical setup.

\section{Results}

We measured solid phantoms with liquid filled capillaries inside that mimic cylindrical tissues with blood vessels, where the liquid volume mimicking the blood is kept constant. The background solution that we measured has a smaller reduced scattering coefficient than the capillaries. In addition, the capillaries contain absorption components. In this phantom model, we are 
able to discern different light intensities throughout the full scattering profile, even though the volume of the components is the same. Our system has the ability to distinguish between different diameters smaller than $1 \mathrm{~mm}$. The distribution of the blood volume inside capillaries with different diameters causes the light to advance in different ways, in accordance to the effective optical properties.
The FSPs of the measured phantoms are presented in Fig. 5. The highest curve represents a phantom that was used as a background tissue, without capillaries, that has a reduced scattering coefficient characteristic to human skin tissue $\left(\mu_{s}^{\prime}=1.04 \mathrm{~mm}^{-1}\right)$ [28]. The other profiles belong to phantoms with capillaries inside, in different diameters: $750 \mu \mathrm{m}$ and $510 \mu \mathrm{m}$, while the volume of the

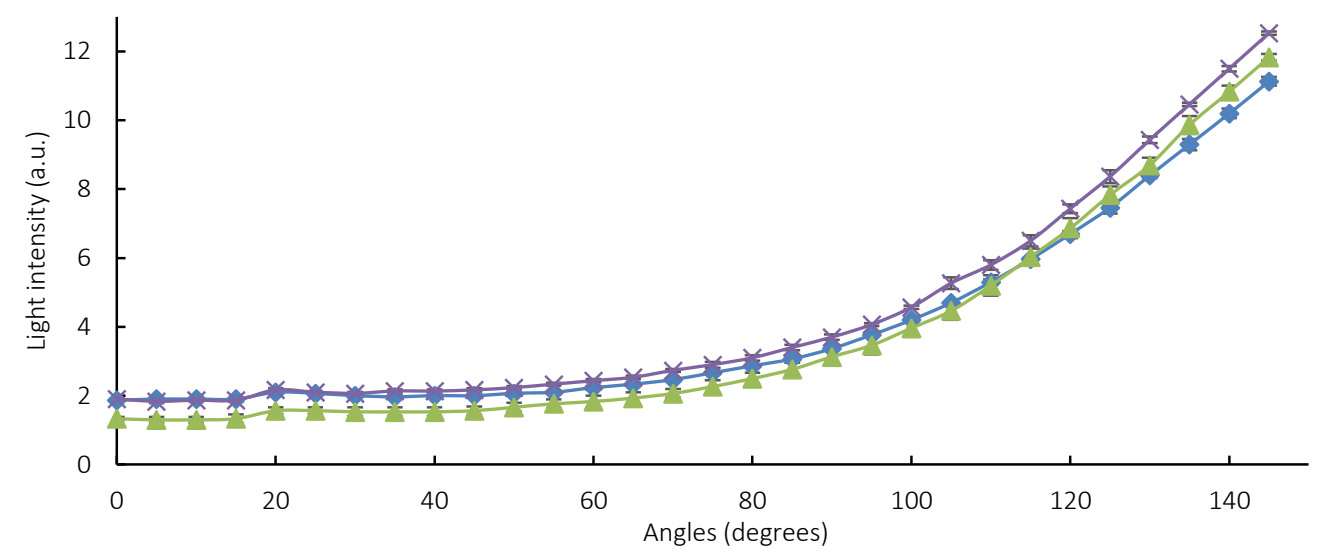

Fig. 5 Full scattering profiles of phantoms in a $13 \mathrm{~mm}$ tube: a background phantom $\left(\mu_{s}^{\prime}=1.04 \mathrm{~mm}^{-1}\right)$ without capillaries (x) and phantoms with capillaries in different diameters (triangles and diamonds for $750 \mu \mathrm{m}$ and $510 \mu \mathrm{m}$ ). The capillaries contain a solution $\left(\mu_{s}{ }^{\prime}=1.04 \mathrm{~mm}^{-1}, \mu_{a}=3 \mathrm{~mm}^{-1}\right)$ in the same volume for each sample.

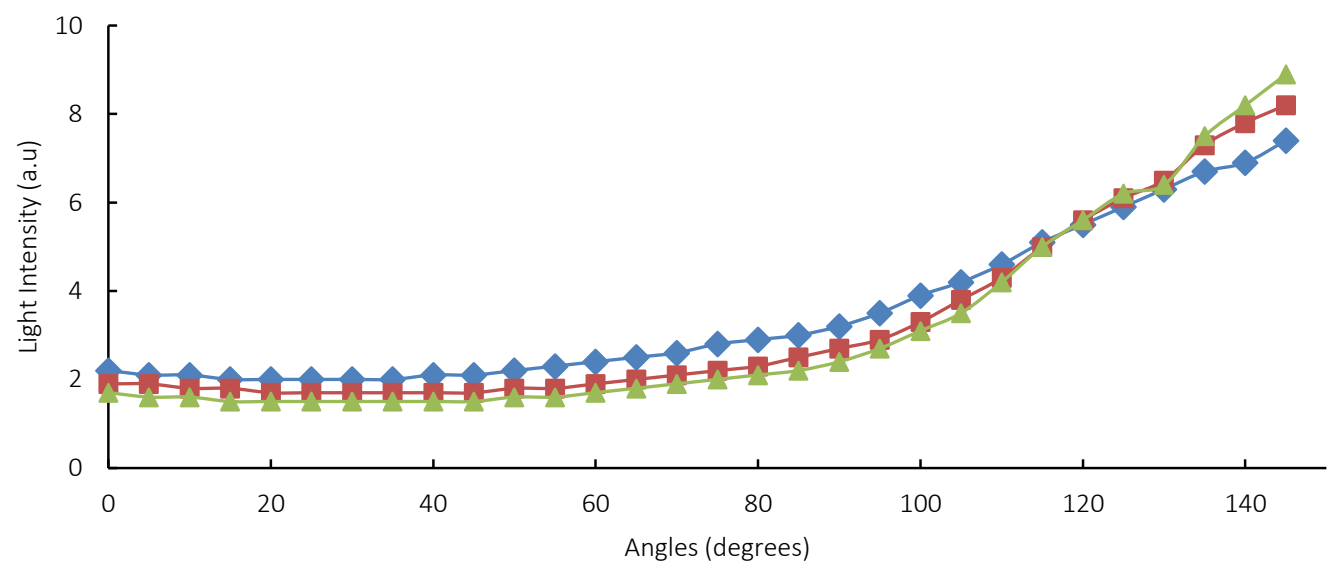

Fig. 6 Full scattering profiles of liquid homogenous phantoms with different reduced scattering coefficients (diamonds, squares and triangles in respect to $1,1.6$ and $2.6 \mathrm{~mm}^{-1}$ ) in a tube of $13 \mathrm{~mm}$ diameter.

blood-like phantom in the capillaries is kept constant (5\%). The isobaric point appears at 115 degrees; at this point a substitution between the curves occurs. The background has no value common with the isobaric point due to the lack of absorbing components within it. The standard deviation is in average lower than $1.5 \%$.

These results correspond with our previous work that presented a linear dependence between the angle of the isobaric point and the cylindrical tissue diameter. There, the isobaric point of $13 \mathrm{~mm}$ homogenous phantoms in different reduced scattering coefficients appeared at $115^{\circ}$, as shown in Fig. 6. The standard deviation is in average lower than $1.5 \%$ also.
The finding of the isobaric point means that the total reduced scattering coefficient of each one of the phantoms is actually different. The measurements of different phantom profiles, that have the same volume of solution in the capillaries and the same background solution, present the shielding effect. While the shielding effect is known as an effect that depends on the absorption of the medium [18-20], in this experiment we mimic blood that has high absorption and also high scattering, and we see the influence of the scattering as well.

In addition, we measured phantoms with constant reduced scattering coefficient, inside the capillaries as 
well as out of them, in addition to the absorption components in the capillaries. The FSPs of these phantoms are presented in Fig. 7 in comparison to the first phantoms. As we expected, the intensities of the FSPs are lower than the FSP of similar phantoms with higher scattering in the capillaries, as a result of the light absorption that is more significant without the high reduced scattering coefficient. The higher standard deviation at the attenuated profiles $(1.8 \%)$, in combination with the subtle change that is expected between the profiles, make it difficult to determine that the absorption alone maintains the profile's format.

(a)

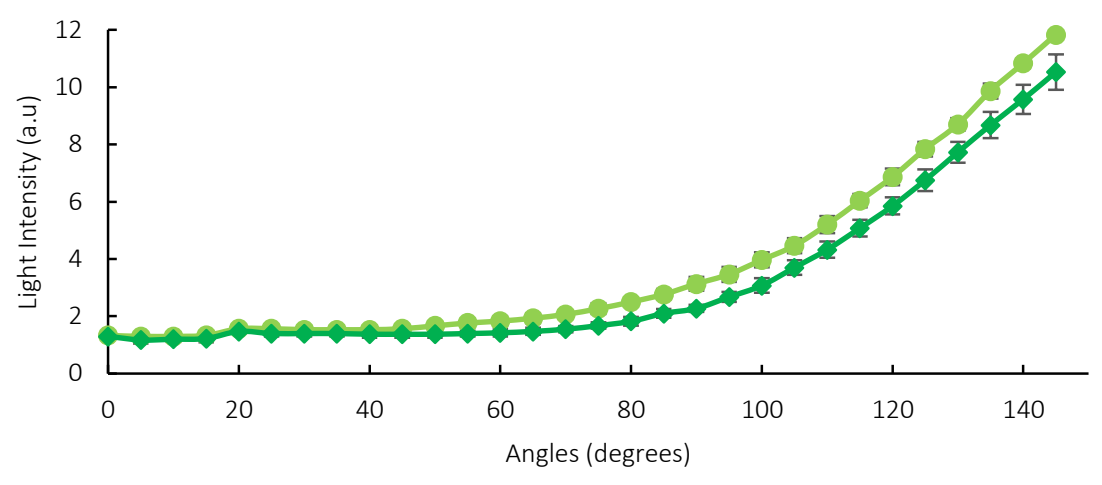

(b)

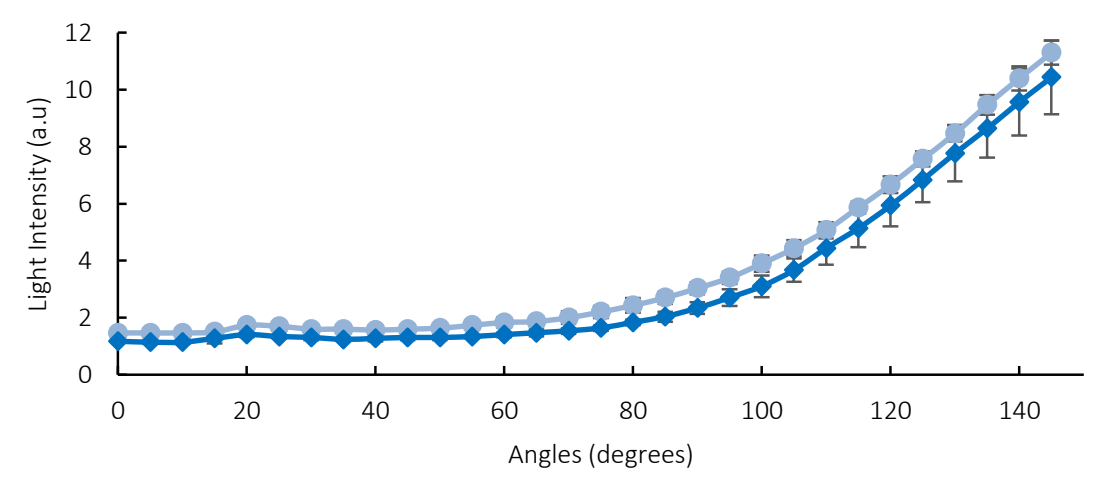

(c)

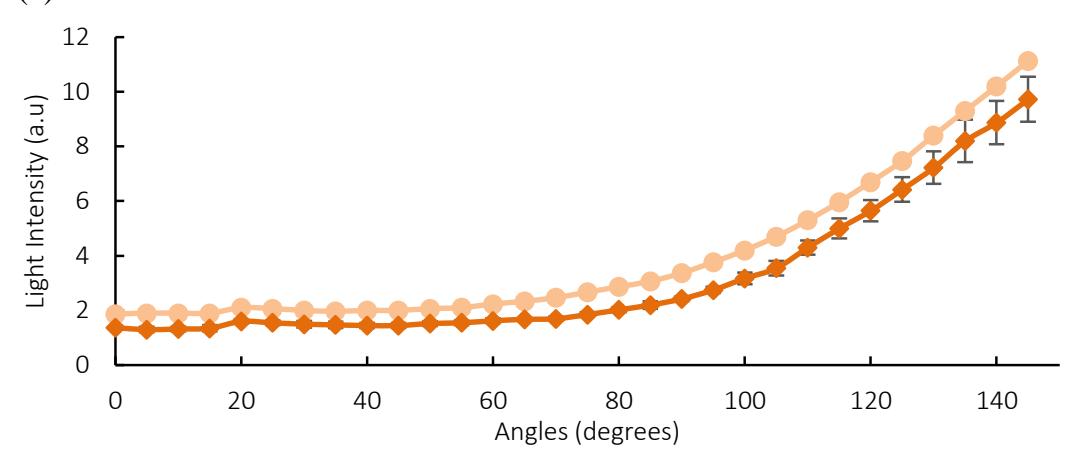

Fig. 7 A distinction of absorption influence within capillaries in different diameters (a) $750 \mu \mathrm{m}$ (b) $600 \mu \mathrm{m}$ and (c) 510 $\mu \mathrm{m}$. FSP of phantom with a lower reduced scattering coefficient in the capillaries $\left(\mu_{s}{ }^{\prime}=1.04 \mathrm{~mm}^{-1}\right)$, which is the same as the background, and absorption coefficient of $\mu_{a}=1 \mathrm{~mm}^{-1}$ (in diamonds) compared to FSP of phantom with a higher scattering coefficient $\left(\mu_{s}^{\prime}=3 \mathrm{~mm}^{-1}\right)$, which is the same as the blood, while the absorption coefficient is the same (in circles).

\section{Discussion}

In the results that we presented above, the order of the profiles in the transmission area (the low angles) is arranged according thus: as the diameter of the capillaries increases, the light intensity decreases (Fig. 8), while in the reflectance area (the high angles) the opposite is true.
In previous experiments [15] we manufactured silicon-based phantoms with blood vessels inside with different diameters larger then $1 \mathrm{~mm}$ (the blood volume remained constant). The obtained STD was smaller than $7 \%$. At the transmission range we could not distinguish between the FSPs of the phantoms, as presented in 
Fig. 9, whereas with the new phantoms with the glass capillaries, we have the ability to discern the changes in the transmission area. However, in the reflection range we obtained the same tendency in both models, above $1 \mathrm{~mm}$ and under $1 \mathrm{~mm}$ - the reflection decreases as the vessel diameter decreases, in accordance with the shielding effect.

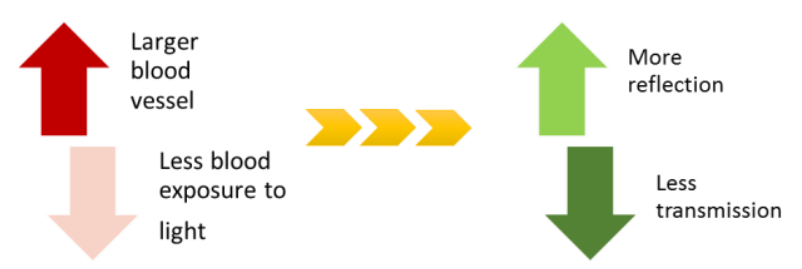

Fig. 8 Tendency of profiles in transmission and reflection area in accordance to blood vessel diameter.

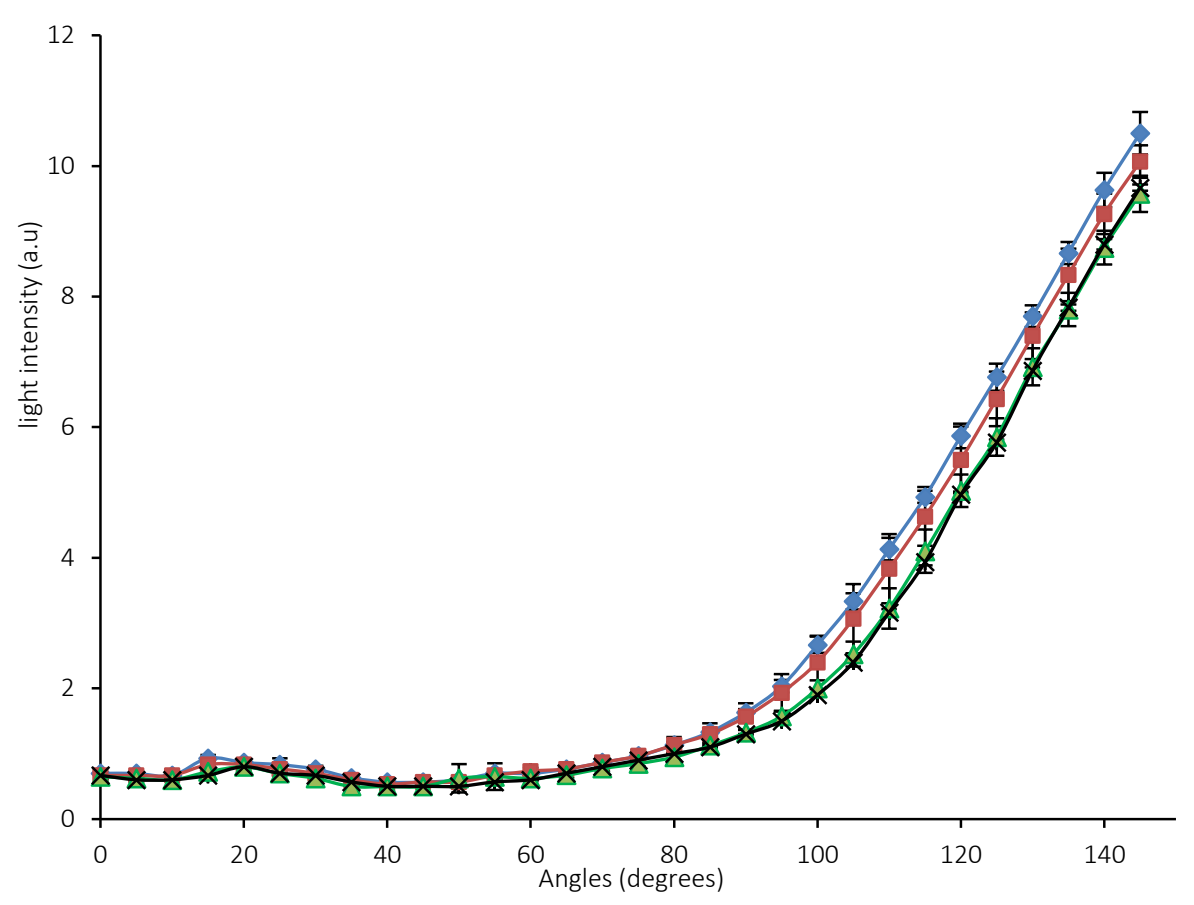

Fig. 9 Full scattering profiles of silicon-based phantoms, $10 \mathrm{~mm}$ diameter. With constant blood volume in different blood vessel diameter (diamonds, squares, triangles and $\mathrm{x}$ for $1.6 \mathrm{~mm} 1.3 \mathrm{~mm}, 1.1 \mathrm{~mm}$ and $1 \mathrm{~mm}$ diameter, respectively). The larger the diameter is, the higher the obtained intensity is.

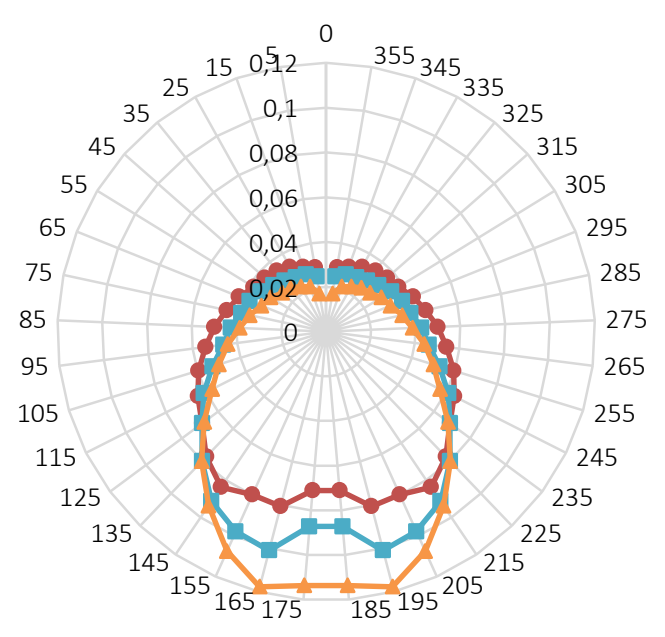

Fig. 10 Radar presentation for simulation results of the full scattering profile of tissue with constant blood volume in vessels in different diameter (circles, squares and triangles indicate 5 vessels of $1 \mathrm{~mm}$ diameter, 40 vessels of $502 \mu \mathrm{m}$ diameter and 200 vessels of $159 \mu \mathrm{m}$ diameter, respectively). Light intensity value (a.u.) is presented at each angle (0 to $360^{\circ}$ ), starts at the center of the chart and ends on the outer ring. The isobaric point appears at $125^{\circ}$. The transmission increases as vessel size increases while the reflection decreases as vessel diameter increases. 
In our simulation, the order of the profiles was in a reversed tendency, as shown in Fig.10. It is important to notice that the scattering definitions in the simulation were higher $\left(4 \mathrm{~mm}^{-1}\right)$, while lower values were determined for the absorption $\left(0.04 \mathrm{~mm}^{-1}\right)$, due to the higher wavelength $(850 \mathrm{~nm})$. In the experiment the impact of the absorption was displayed more dominantly and therefore the profiles' order was inverse. Since the absorption causes the attenuation of the whole profiles' intensities, as we showed in a previous work [12], we can expect to obtain more attenuation for smaller vessels with stronger effectivity, as a result of the shielding effect. Therefore, the intensities in the reflectance area increase as the vessel diameter increases. The smaller the vessel is, the stronger the obtained effective absorption, hence the effective scattering coefficient of the whole phantom is decreased. However, in the transmission area we obtain an opposite gradation for the curves.

According to our previous research [12,29], based on homogenous phantoms with different reduced scattering coefficients, it can be concluded that the curves' gradation is in accordance with the tendency of the reduced scattering coefficient, as shown in Fig. 11. In the transmission range (low angles) the light intensity of higher $\mu_{\mathrm{s}}$ ' is weaker, while in the reflectance range (high angles), for higher $\mu_{\mathrm{s}}$ ' the intensity is stronger. The many scattering events prevent photons from advancing toward the transmission region and cause them to reach the reflectance area. On the other hand, for lower $\mu_{\mathrm{s}}^{\prime}$ the opposite occurs, because less scattering events occur. Hence, we can conclude in this capillaries experiment, that larger diameter of capillaries yields a medium with a higher total reduced scattering coefficient than the smaller diameter, due to the less effective absorption there. However, with smaller capillaries the absorption is more expressed.

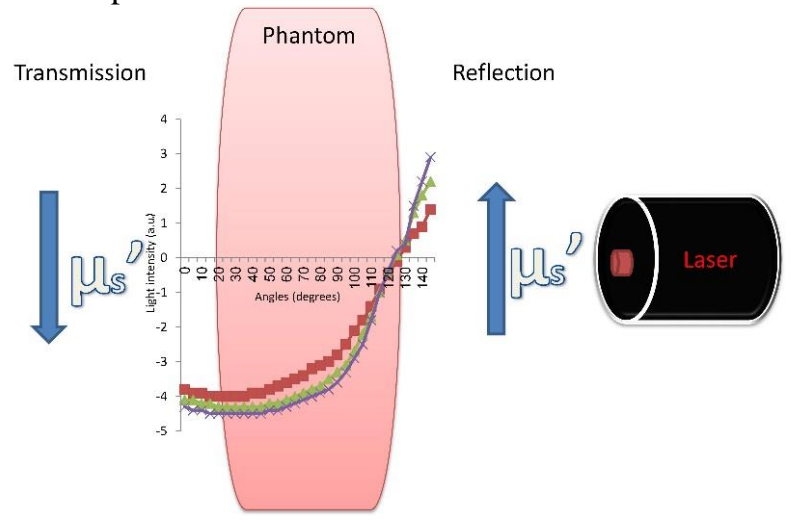

Fig. 11 The trend of the reduced scattering coefficient in accordance with the range.

\section{Conclusion}

In this research, we present the role of the blood vessel diameter in light-tissue interactions using our model of FSP. We prepared cylindrical phantoms with glass capillaries in different diameters (smaller than $1 \mathrm{~mm}$ ), and they were filled with blood-like solution. The FSP that we measured experimentally corresponded with our simulation results. We found the isobaric point, which is non-dependent on the optical properties. The angle where the isobaric point appeared in the experiment is $115^{\circ}$, while in the simulation it appeared at higher angle, probably due to the assumptions which made for the simplification of the simulation. The results that we present here validate that the isobaric point overcomes the shielding effect. The importance of this finding is that this unique point can be useful as a reference point for clinical purposes. For example, it can be implemented in various optical methods such as NIR spectroscopy and PPG experiments, and thus can improve analyzing oxygen saturation values, blood perfusion and blood pressure. In addition, it is relevant as well to the development and promotion of imaging and tomography methods, owing to the new viewpoint of FSP. On the one hand, the FSP method has the ability to distinguish between minor changes in the blood vessel diameter, and on the other hand it has the uniqueness of the fixed isobaric point, serving as a self-reference point. 